\title{
Voix de femmes
}

Témoignages de jeunes filles juives grecques survivantes de la Shoah

Women's Voices: Testimonies of Greek Jewish Women Who Survived the Shoah

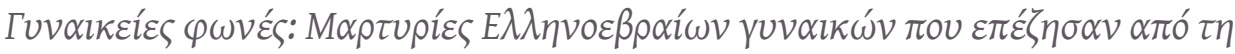
Shoah

Odette Varon-Vassard

\section{OpenEdition}

1 Journals

Édition électronique

URL : https://journals.openedition.org/ceb/8528

DOI : $10.4000 /$ ceb.8528

ISSN : 2261-4184

Éditeur

INALCO

Édition imprimée

ISBN : 978-2-85831-229-0

ISSN : 0290-7402

Référence électronique

Odette Varon-Vassard, « Voix de femmes », Cahiers balkaniques [En ligne], 43 | 2015, mis en ligne le 25 juillet 2017, consulté le 06 juillet 2021. URL : http://journals.openedition.org/ceb/8528 ; DOI : https:// doi.org/10.4000/ceb.8528

Ce document a été généré automatiquement le 6 juillet 2021.

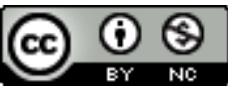

Cahiers balkaniques est mis à disposition selon les termes de la Licence Creative Commons Attribution - Pas d'Utilisation Commerciale 4.0 International. 


\title{
Voix de femmes
}

\author{
Témoignages de jeunes filles juives grecques survivantes de la Shoah \\ Women's Voices: Testimonies of Greek Jewish Women Who Survived the Shoah

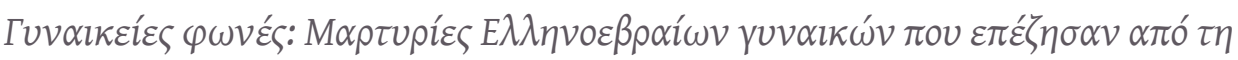 \\ Shoah
}

Odette Varon-Vassard

À la mémoire de Berry Nahmias

1 Quelques mots seulement en guise d'introduction ${ }^{1}$. Comme ma communication est la seule du colloque qui concerne la mémoire de Juifs survivants de la Shoah, je ferai en premier lieu référence à l'émergence difficile et tardive de cette mémoire en Europe et surtout en Grèce². Par la suite, vu que j'ai suivi le premier axe qui nous était proposé dans le colloque et qui concernait exclusivement les témoignages de femmes, je me suis focalisée sur un petit corpus de trois témoignages de jeunes filles juives grecques déportées à Auschwitz-Birkenau et ayant survécu à la Shoah. Je ne vais pas m'arrêter ici sur les lieux communs des récits des déportés (qu'ils soient hommes ou femmes) : à savoir l'épreuve du voyage, l'arrivée au camp, l'apprentissage d'un quotidien totalement différent dans des conditions inhumaines, ce que Tzvetan Todorov a appelé «face à l'extrême " ${ }^{3}$. J'essaierai par contre de faire ressortir certaines spécificités du discours féminin et même de la condition féminine dans les camps de la mort.

\section{L'émergence d'une mémoire difficile et tardive}

2 Parlons tout d'abord de la mémoire, c'est-à-dire des témoignages. La même décennie qui a vu la recherche historique débuter autour du sort des Juifs de Grèce sous l'Occupation (les années 1990) marque également l'époque des témoignages en Grèce, qu'ils soient écrits ou oraux. Les raisons de ce retard sont nombreuses. Outre qu'aucun éditeur ne se serait jamais hasardé à publier plus tôt de tels textes, considérant qu'ils ne présentaient aucun intérêt, la plupart des survivants n'étaient prêts ni à parler ni à 
écrire. Certaines de ces raisons sont les mêmes que celles que Jorge Semprún, déporté à Buchenwald pour actes de résistance, a si bien décrites et analysées dans l'Écriture ou la $v i e^{4}$. Jorge Semprún raconte pourquoi il lui était impossible d'écrire pendant la première quinzaine d'années qui a suivi son retour des camps. En effet, l'écriture renvoyait à la mort et il avait choisi, lui, la vie. Ce n'est que plus tard, lorsque l'expérience du camp s'est imposée à lui, qu'il n'a pas abandonné cette thématique essentielle jusqu'à la fin de sa vie.

3 Mais un grand silence entoure aussi la question pendant les premières décennies d'après-guerre. "L'indicible", selon l'expression utilisée pour caractériser cette expérience-limite pour les survivants, était aussi « inaudible » car les premiers qui ont essayé d'en parler se heurtaient à un mur de silence et d'indifférence. Les sociétés et les individus n'étaient pas prêts du tout à entendre "ces délires de fous ", comme on l'a cru de prime abord. Ce n'est qu'au début des années 1960, avec le procès Eichmann en Israël, pendant lequel 111 survivants ont parlé pour la première fois, que la voix des victimes a commencé à se faire entendre. C'est ce même procès, d'ailleurs, qui a donné naissance au livre si important et en même temps si controversé de Hannah Arendt: Eichmann à Jérusalem: rapport sur la banalité du mal5. Dans l'Ère du témoin, Annette Wieviorka note à propos de ce procès :

Le procès Eichmann marque un véritable tournant dans l'émergence de la mémoire du génocide en France, aux États-Unis comme en Israël. Avec lui s'ouvre une ère nouvelle: celle où la mémoire du génocide devient constitutive d'une certaine identité juive tout en revendiquant fortement sa présence dans l'espace public ${ }^{6}$.

\section{Témoignages de Juifs grecs}

4 Je commence par un survol bibliographique. La première édition qui recueille des témoignages de Juifs Grecs n'est pas une édition grecque. Il s'agit d'un volume francophone, publié à Paris en 1967 sous le titre le Passage des Barbares, qui est seulement traduit en grec en 1985 (trois ans après la reconnaissance de la Résistance nationale par le gouvernement socialiste d'Andréas Papandréou). Miriam Novitch, ellemême survivante du camp d'Auschwitz et collaboratrice des archives israéliennes Lohamei Hagetaoth (kibboutz fondé par les survivants du ghetto de Varsovie), a mené la recherche et recueilli les entretiens ${ }^{7}$. L'auteur a rassemblé une très importante masse de données, constituée surtout de témoignages de Juifs grecs (hommes et femmes) ayant survécu aux camps de la mort ou ayant participé à la Résistance. J'insiste sur le fait que Miriam Novitch a inclus les témoignages de Juifs résistants, parce que ce sujet très important commence à peine à être étudié (on trouve quatre noms de femmes parmi les Juifs résistants grecs). Ce sujet revêt une importance prépondérante, car il contrecarre le mythe de la " passivité » que certains historiens attribuent aux Juifs au moment de la déportation, et il dénote aussi un plus grand degré d'intégration des Juifs dans la société hellénique. La traduction grecque, faite en 1985, a été éditée par une association à but non lucratif. Le livre n'étant pas disponible dans le commerce, il a eu un caractère "confidentiel» et a été réservé essentiellement, lui aussi, aux bibliothèques des familles juives qui restaient encore très francophones. Je dis « lui aussi » en pensant au livre du rabbin Michaël Molho et de l'historien Joseph Nehama, In memoriam (1948), qui a connu un sort éditorial analogue. Il a fallu attendre encore quarante ans pour que le sujet de la participation des Juifs grecs au sein de la Résistance intéresse à nouveau le milieu de la recherche universitaire. Le sujet a refait 
surface quand Steven Bowman, professeur d'histoire à l'université de Cincinnati, a publié en 2006 l'ouvrage intitulé : Jewish Resistance in Wartime Greece. La traduction en grec de ce livre a paru en 2013 et a été effectuée par les soins du Conseil central Israélite de Grèce (KISE). Paru en 2014, le livre de Rika Benveniste consacre une large partie à l'étude d'un groupe de jeunes saloniciens qui ont rejoint le maquis de l'ELAS'.

5 À Athènes, le Musée Juif de Grèce a inauguré en avril 2013 la première exposition sur ce sujet, sous le titre : Synagonistis ${ }^{9}$. Cette thématique a été la dernière à être explorée dans les expositions de ce musée. L'exposition itinérante sur la Shoah organisée par ce musée, qui a voyagé à Salonique, Strasbourg et en d'autres villes, a eu lieu en 2000. Quant à l'exposition sur les enfants cachés, elle a eu lieu en 2003. Cela coïncide avec le cheminement de la prise de conscience collective: d'abord, la Shoah a monopolisé l'intérêt de la recherche; bien après, on s'est intéressé aux " enfants cachés » (terme est internationalement reconnu en 1990). Longtemps après, on s'est penché sur les Juifs grecs ayant pris part à la Résistance.

Pourtant, l'histoire des Juifs sous l'Occupation ne peut être complète que si elle comporte ces trois volets : déportation (et extermination pour l'écrasante majorité), résistance et sauvetage (d'adultes et d'enfants). Ce n'est pas un hasard si le film de Vassilis Loules Baisers aux enfants [ $\Phi_{l} \lambda_{l} \alpha \dot{\alpha} \varepsilon l \varsigma \tau \alpha \pi \alpha l \delta i \alpha ́$ ], inspiré par l'exposition du musée sur les enfants cachés, et qui recueille les témoignages de cinq enfants Juifs grecs cachés et sauvés pendant la guerre, est sorti en 2012 et a connu un franc succès depuis lors en Grèce et à l'étranger ${ }^{10}$. Le réalisateur aborde avec une grande sensibilité un domaine totalement vierge pour le cinéma grec: le domaine de «ceux qui n'avaient rien à raconter ", pour reprendre l'expression ironique du psychiatre et psychanalyste Boris Cyrulnik, lui-même ancien enfant caché ${ }^{11}$.

7 Pour revenir aux témoignages des survivants. Pendant les années 1990, une « explosion éditoriale » de témoignages de survivants a rompu le silence qui avait duré entre quatre et cinq décennies. L'édition des témoignages des Juifs grecs suit avec un certain décalage la publication de tels témoignages en Europe. L'activité éditoriale en Europe occidentale commence en effet au lendemain de la Libération avec "les livres du souvenir» au sein des survivants de la communauté juive ashkénaze, comme nous l'apprend Annette Wieviorka ; elle se développe dans les années 1960 et 1970 et explose dans les années 1980.

Jorge Semprún note pour les années 1960 :

Comme si au-delà de toute circonstance biographique, une capacité d'écoute avait mûri objectivement, dans l'opacité quasiment indéchiffrable des cheminements historiques. Mûrissement d'autant plus remarquable et passionnant qu'il coïncide avec les premiers témoignages sur le Goulag soviétique ${ }^{12}$.

9 Jorge Semprún se réfère au livre d'Alexandre Soljenitsyne qui paraît en Occident à la même époque et qui contribue à démolir définitivement sa foi déjà très ébranlée dans le communisme. Ainsi se dessine le champ particulier de la littérature concentrationnaire, mais ceci est une autre histoire.

Sur le plan des témoignages, la Grèce a accumulé un retard important puisque la plupart des témoignages ont été édités à partir de la fin des années 1980 et surtout pendant les années $1990^{13}$. De 1981 à aujourd'hui ont paru en Grèce environ vingt-cinq témoignages écrits (en grec), la plupart de survivants originaires de Salonique, surtout des hommes, le corpus de témoignages de femmes étant nettement plus faible. De plus, un volume recueillant des témoignages oraux de survivantes et survivants juifs de 
Salonique a paru en $1998^{14}$. Il comportait vingt-cinq témoignages d'hommes et vingt-etun témoignages de femmes ayant entre 12 et 28 ans au moment de leur déportation. C'est tout ce qui reste comme trace écrite de la florissante «mère d'Israël » ou de la "Jérusalem des Balkans", comme on appelait alors Salonique. Rappelons que cette ville, qui a perdu à peu près 46000 habitants pendant la Shoah (sur les 49000 de sa population juive d'avant-guerre, recensement de 1940), abritait de loin la plus importante communauté juive de Grèce. C'est ce qui a conduit André Séphiha à parler de " désert du Sahara » à son retour à Salonique, après avoir fui avec sa famille dans le Péloponnèse de septembre 1943 à octobre $1944^{15}$.

11 Je voudrais aussi relever l'importance de certains témoignages de survivants provenant d'autres villes, comme celui de Berry Nahmias ${ }^{16}$, originaire de Kastoria, $d^{\prime}$ 'Errikos Sevillias ${ }^{17}$ originaire d'Athènes et de Nata Osmo-Gattegno ${ }^{18}$, originaire de l'île de Corfou. Une édition bilingue du Conseil central Israélite de Grèce (KISE), financée par le Secrétariat Général pour la Jeunesse, à but pédagogique surtout, a vu le jour en 2008. Dans ce volume on a repris des extraits de témoignages écrits et on a recueilli des témoignages oraux de trois catégories de Juifs grecs : des survivants des camps, des résistants et des enfants cachés. Ce livre donne ainsi une image plus complète du sort des Juifs grecs pendant l'Occupation. Chaque partie est accompagnée d'un texte introductif ${ }^{19}$. L'édition avait pour but d'entrer dans les bibliothèques des écoles publiques et son financement était un geste important de la part de l'État grec.

12 Je vais par la suite me focaliser sur le corpus de témoignages de femmes juives grecques survivantes des camps.

\section{Les mémoires de Lisa Pinhas}

On pensait que l'ère des témoignages écrits était révolue mais les «fonds de tiroirs » nous surprennent encore. Un dernier (jusqu'à présent) témoignage écrit vient en effet d'être édité en 2014 par le musée juif de Grèce car le manuscrit appartient à ses collections ${ }^{20}$. La famille de la défunte l'a confié plusieurs années auparavant à ce musée pour qu'il soit édité. Pourquoi Lisa Pinhas n'a-t-elle pas voulu ou pas pu l'éditer ellemême? D'abord parce que dans les années 1970 il était encore trop tôt pour les témoignages des survivants juifs en Grèce, même si l'on sait par la famille que Lisa Pinhas s'était mise à rédiger son témoignage dès son retour des camps. Je considère que le fait qu'il ait été rédigé en français constituait aussi un obstacle. Mais à mon avis, c'était surtout le fait qu'elle-même ne pouvait pas arrêter de le reprendre et le perfectionner.

Elle a cependant donné des conférences de son vivant ; elle a représenté les survivants juifs grecs et elle a effectué très tôt le voyage de retour à Auschwitz. Autrement dit, on peut affirmer que c'est une femme qui a servi par son activité la cause de cette mémoire et, d'habitude, des femmes comme Erika Kounio-Amariglio ou Berry Nahmias recueillent aussi les témoignages des autres et publient leur propre témoignage. Ces trois femmes constituent des figures emblématiques de la mémoire de la Shoah en Grèce, ayant œuvré beaucoup pour la reconnaissance de cette mémoire.

Les spécificités du témoignage de Lisa Pinhas, sépharade de Salonique, sont nombreuses. Tout d'abord, ce témoignage s'inscrit dans le très faible corpus de témoignages écrits par des femmes et l'enrichit considérablement. En outre, il est rédigé dans un français impeccable, fruit des années d'études de l'écrivaine dans les 
grands établissements francophones de l'Alliance Israélite Universelle à Salonique, qui étaient très fréquentés par les Juifs des classes moyennes, mais aussi par des enfants des classes pauvres.

Lisa Pinhas, qui a perdu dans les camps cent douze membres de sa famille ainsi que son mari, a commencé à rédiger son témoignage, comme on l'a déjà dit, dès son retour du camp. Peut-être les détails du quotidien étaient-ils encore, à ce moment-là, extrêmement vivaces dans sa mémoire. C'est la raison pour laquelle elle a pris la plume «à chaud». Elle n'a pas cessé de travailler et a perfectionné ce manuscrit jusqu'au début des années 1970 lorsqu'un accident cérébral l'empêcha de continuer. Le fait qu'elle n'ait pas attendu le "second temps", l'ère des témoignages, a peut-être condamné son manuscrit à dormir au fond d'un tiroir pendant de longues années. Ce témoignage précoce qui devait inaugurer la publication des témoignages des Juifs grecs maintenant est très probablement le dernier édité (il clôt pour le moment le corpus).

Il s'agit en tout cas d'un des témoignages les plus riches et les plus précis. Il a la forme et la qualité d'un vrai livre, ce qui n'est pas toujours le cas des autres témoignages. Dans le cas des témoignages oraux, plusieurs témoins ont raconté ce qu'ils ou elles pouvaient raconter et la transcription ne couvre que quelques pages. Par ailleurs, la démarche des personnes qui écrivent est totalement différente de celles qui répondent à une interview. La volonté de laisser un témoignage complet est commune à ce type de projet. Mais ce qui frappe de prime abord dans le livre de Pinhas - car il s'agit d'un livre très bien construit - c'est la structure, la précision, et la façon très détaillée de relater le quotidien d'une détenue juive dans le camp d'Auschwitz-Birkenau. Ainsi, nombre d'éléments qu'on connaissait déjà autour du quotidien des camps (nourriture, habillement, maladies, conditions et dureté de la vie) s'éclaircissent jusque dans les moindres détails. Le talent de l'écrivaine, qui est incontestable, ne laisse pas le lecteur s'ennuyer un seul instant, lorsqu'il lit les deux cent vingt-deux pages très denses du tapuscrit.

Lisa Pinhas a conscience de la spécificité féminine de son témoignage car elle commence son récit en ces termes: "À l'instar de plusieurs autres qui ont écrit quelques pages sur les camps de concentration des hommes, je veux à mon tour vous donner quelques détails sur ceux des femmes, tels que je les ai vécus». On peut ici parler d'un véritable univers concentrationnaire au féminin. Dans un témoignage de femme, il ne s'agit jamais uniquement, en outre, d'une histoire personnelle. Chacune des survivantes parle aussi des autres qui ont péri (sœurs, cousines, camarades).

Charlotte Delbo, déportée politique dont le convoi a été conduit pour des raisons tout d'abord inconnues à Auschwitz, a poussé l'intérêt et l'attachement pour ses camarades jusqu'à leur consacrer un livre entier, dans lequel elle a reconstitué, des années après les faits, la biographie de chacune d'elles ${ }^{21}$. Je considère qu'une première spécificité des témoignages féminins est la très grande place conférée aux autres. D'ailleurs, la notion d'une solidarité féminine supérieure à la solidarité masculine comme condition de la survie des détenues des camps est une hypothèse déjà émise dans la bibliographie internationale, comme le confirme l'universitaire grecque Franguiski Abatzopoulou ${ }^{22}$.

\section{La solidarité}

Dans le livre de Pinhas, les autres, ce ne sont pas seulement les camarades qui ont péri, ou une amie chère. L'auteure se réfère à une multitude de femmes, toujours désignées 
par leur prénom et par leur nationalité (dans l'univers juif du camp les identités nationales reprennent le dessus), au point que l'on peut dire que la sociabilité occupe une place prépondérante dans cette œuvre. Cela m'incite à dire que la sociabilité a été aussi une condition de survie (je n'aime pas beaucoup le terme de "stratégie», qui induit une réflexion rationnelle et qui comporte une forte nuance d'arrière-pensée). Pinhas se réfère parfois aux autres détenues de façon telle qu'on a l'impression de les voir mener une vie normale. Je cite à titre d'exemple :

Pour mon compte j'avais fait la connaissance d'une jeune polonaise qui s'appelait Hanka. Elle travaillait à la cuisine. C'était une jeune femme cultivée, intellectuelle. Nous nous entendions à merveille [en français évidemment, complicité de femmes cultivées] et une amitié des plus sincères nous lia très vite. Mes petites amies Bella et Mini et ma jeune sœur Marie et moi, nous lui apportions journellement des bijoux de grande valeur, de l'argent, du beau linge et des bas fins, du savon parfumé, etc. Il n'existait pas entre nous ce vulgaire marchandage comme chez les autres. Nous lui offrions tout et elle nous donnait des provisions sans discontinuer (p. 73. Cette numérotation de page renvoie au manuscrit).

Plus loin, elle nous dit que quand elle ne travaillera plus au Kommando « Kanada » (le Kommando chargé de récupérer et de trier la montagne de vêtements et d'effets personnels laissés par les déportés à leur arrivée au camp) et qu'elle n'aura donc plus rien à lui offrir, Hanka ne cessera de lui apporter la nourriture indispensable pour sa survie.

Nata Osmo-Gattegno, originaire de la vieille communauté juive de l'île de Corfou, écrit que les jeunes filles juives déportées de son île sont restées unies jusqu'à Bergen-Belsen, sauf celles qui sont restées à Birkenau pour y être exterminées. La communauté juive de Corfou $^{23}$ a été entièrement déportée (2000 personnes environ). Elle ne compte que de très rares survivants, ce qui rend précieux cet unique témoignage. Nata Osmo-Gattegno évoque systématiquement le groupe des jeunes filles de Corfou. $\mathrm{Ne}$ pas se couper d'un groupe, ne pas se sentir totalement agressée sans perdre tous ses repères, était aussi une condition de survie. Elle raconte :

Il y avait un groupe de jeunes filles de Corfou qui chantaient très bien. Elles s'asseyaient à un endroit et elles chantaient. Les autres grecques me protégeaient, car la responsable cherchait des prétextes pour m'agresser (p. 100).

Elle évoque aussi une fille qui l'a sauvée alors qu'elle était gravement malade :

Une jeune fille, Rachel Vital-Ouziel, venait systématiquement me rendre visite. [...] On la battait pour ça, mais elle continuait de venir. Un jour, elle a attrapé un matelas, elle l'a porté au-dessus de sa tête et elle a essayé de venir me voir [...]. Mes amies ont été près de moi et m'ont beaucoup aidée à cette époque-là (p. 104).

Nata Osmo-Gattegno a beaucoup aidé les autres. On peut dire qu'elle avait un souci constant des autres prisonnières : elle pouvait leur redonner du courage, partager son pain avec elles ou leur apporter des provisions, au péril de sa propre vie.

Les quatre filles de la famille Osmo ont connu chacune un sort différent. Mais pour comprendre l'histoire de la famille, il faut d'abord prendre en compte ce qui a constitué le drame familial avant la guerre : la perte du fils aîné, mort de maladie dans son adolescence. Nata, qui est la première-née après la naissance du frère, va dorénavant, à mon avis, prendre le rôle du frère, de celui qui doit protéger les parents et les trois sœurs qui sont nées après elle. Cela va être décisif pendant l'Occupation pour le sort de chacune. Nata va devenir très vite membre de l'organisation de Résistance des Jeunes l'EPON $^{24}$. Avec l'aide de l'EPON elle pourra assurer la fuite des deux sœurs suivantes 
vers les villages, tandis qu'elle-même ne quittera jamais ses parents. Ainsi les deux sœurs ne seront pas déportées, tandis qu'elle suivra ses parents et sa plus jeune sœur et sera déportée avec toute la communauté de Corfou à Auschwitz. Dans ce cas, la déportation a été un choix et pas un sort subi. Nombre de jeunes gens ont pris la même décision pour ne pas quitter des parents et des frères et sœurs, élément qui doit relativiser les accusations de " passivité » portée à l'encontre de ces jeunes.

Les deux sœurs ont épousé des jeunes Corfiotes chrétiens orthodoxes et se sont converties. L'une des deux a réussi à trouver le bonheur et à avoir un enfant. L'autre a été rongée par le remords et est tombée gravement malade. Nata va retrouver ses sœurs lorsqu'à la fin de la guerre elle reviendra à Corfou. Elle a eu plusieurs possibilités de mariage sur place, mais elle s'est rendue en Israël où elle a finalement épousé le jeune Israël Gattegno (lui aussi Grec d'origine juive). Ils ont vécu heureux jusqu'à leur vieillesse, mais n'ont pas pu avoir d'enfants, car Nata était devenue stérile pendant sa captivité en camp de concentration ${ }^{25}$.

La quatrième et plus jeune sœur (encore adolescente) a été déportée et exterminée avec les parents dès son arrivée au camp. Chacune des quatre filles de la même famille a donc connu un sort différent, soit en raison du hasard (Nata était plus âgée, donc sélectionnée parmi les prisonnières aptes au travail), soit en raison d'un psychisme différent. Nata a eu l'occasion de s'évader à quatre reprises pendant le transport au camp. Elle a toujours choisi de rester pour ne pas quitter ses parents et sa petite sœur. Elle doit sa survie en partie à la chance et en partie à sa force morale et son dévouement aux autres, qu'elle n'oubliait jamais.

Erika Kounio-Amariglio note un jour différent dans sa vie de prisonnière :

26 mars 1944, un jour comme tous les autres, mais un jour différent pour moi personnellement. C'est le jour où j'ai senti profondément ce que veulent dire les mots amour, fraternité, solidarité et ingéniosité, contre tous ces tyrans. Dans l'enfer c'est un rayon de soleil ! J'ai mon anniversaire et mes amies n'ont pas voulu que cela passe inaperçu! Lorsque nous sommes rentrées le soir du travail, j'ai trouvé sur mon lit une petite fleur et différents objets tout autour. Le gâteau était constitué de deux tranches de pain avec de la confiture et de la margarine (p. 111).

Cette «fête d'anniversaire » revêtait également la forme d'acte de résistance dans le contexte donné.

Je pourrais multiplier les exemples, mais je m'arrête pour dire que des témoignages de moments pareils prouvent que, même "face à l'extrême", il y avait des brèches d'humanité. Ce qui répond à la question de Primo Levi Si c'est un homme/si c'est une femme posée dans le poème en exergue de son premier livre : ceux et celles qui avaient réussi à ne pas perdre totalement leur humanité, avaient sans aucun doute plus de chances de survivre ${ }^{26}$. La résistance humaine était telle que ces jeunes détenu(e)s pouvaient être encore, par moments, des hommes et des femmes, prouvant ainsi, avec Robert Antelme, que, contrairement à toutes les apparences et au but des nazis, ils/ elles appartenaient encore à l'espèce humaine ${ }^{27}$ dont tout voulait les exclure. La solidarité est une forme d'humanité par excellence. C'est sans doute à cause de cela qu'elle était si strictement interdite et si sévèrement punie.

\section{Le sentiment d'identité nationale}

Les sentiments d'identité nationale devenaient plus forts dans les camps. Dans leur univers clos, les détenus juifs se démarquaient par leur identité nationale, et ces 
identités, dont les nazis voulaient absolument les priver en les réduisant uniquement au statut du Juif, reprenaient pour eux le dessus. Pinhas consacre tout un chapitre aux "Hongroises ». Primo Levi parle longuement dans la Trêve des Grecs de Salonique, qui lui ont fait une très forte impression ${ }^{28}$. On connaît des témoignages qui parlent de moments où les détenus chantent avec émotion l'hymne national de leur pays d'origine ou des chansons de leur pays natal.

Mais Pinhas raconte une histoire différente. Je la cite :

À l'approche des fêtes de fin d'année, les Allemands engageaient une poignée de détenues parmi les plus adroites et les occupaient à fabriquer des joujoux pour l'arbre de Noël. Madame Cohen et Paulette, une autre petite Française, m'apportaient des brins de laine de plusieurs couleurs avec quoi je m'évertuais à faire de petits evzones que j'offrais à mes camarades hommes ou femmes (p. 151).

Elle continue en décrivant minutieusement les vêtements et les chaussures ainsi que l'émotion des Juifs grecs qui les voyaient. J'ai trouvé émouvant ce paradoxe : les petites figurines des evzones (ou tsoliades) comme symbole de résistance et d'appartenance nationale dans le camp d'Auschwitz, tandis qu'en Grèce à la même époque, les vrais evzones appartenaient à des groupes de collaborateurs ${ }^{29}$.

Ces jeunes grecques juives refusent que leur identité soit uniquement associée à leur condition de juives, ce à quoi les nazis voulaient les réduire ( tu es Juif, donc tu n'es rien d'autre »).

Berry Nahmias, disparue en 2013, et ayant consacré presque trente ans à servir la mémoire de la Shoah par toutes sortes d'activités, éprouvait le même amour pour sa ville natale : Kastoria. D'ailleurs, elle consacre plusieurs pages dans son témoignage à la description de sa douce vie d'avant la déportation. Dans son témoignage elle parle aussi des chansons grecques que les jeunes détenues chantaient.

Quant à Nata Osmo-Gattegno, longtemps après s'être installée en Israël, sa nouvelle patrie, elle finit son témoignage sur ces mots :

Je n'oublie jamais ma terre natale bien aimée, Corfou. [...] Je n'oublie jamais le coucher du soleil à Pélekas. Je crois que c'est le plus beau du monde (p. 149).

37 Je vais devoir m'arrêter ici tout en sachant que je n'ai fait qu'effleurer les trésors de ces témoignages. Ils restent des sources intarissables pour l'approche de la condition humaine dans les camps nazis.

\section{BIBLIOGRAPHIE}

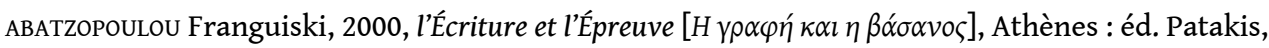
p. 286-337.

ANTELME Robert, 1957, l'Espèce humaine, Paris : Gallimard.

ARENDT Hannah, 1997, Eichmann à Jérusalem, 2e édition augmentée, Paris : Gallimard, « Folio ». BENVENISTE Rika, 2014, Ceux qui ont survécu : résistance, déportation, retour, Athènes : Polis (en grec). 
CYRULNIK Boris, 2010, Je me souviens..., Paris : Odile Jacob.

CYRULNIK Boris, 2012, Sauve-toi, la vie t'appelle, Paris : Odile Jacob.

DELBo Charlotte, 1961, le Convoi du 24 Janvier, Paris : Éditions de Minuit.

KOUNIO-AMARIGLIO Erika, NAR Albertos, 1998, Témoignages oraux des Juifs de Salonique sur la Shoah, Salonique : éd. Paratiritis.

LEVI Primo, 1987, Si c'est un homme, Paris : Julliard.

LEVI Primo, 2003, la Trêve, Paris : Librairie générale française, « Le livre de poche ».

NAHMIAS Berry, 1989, Cri pour le lendemain, Athènes : éd. Kaktos.

Novitch Miriam, 1982, le Passage des barbares : contribution à l'histoire de la déportation et de la résistance des Juifs de Grèce, $2^{\mathrm{e}}$ édition, Israël : Ghetto Fighters House Publishers, [ $1^{\text {re }}$ éd. en français, 1967, Paris : Presses du Temps Présent].

oSMO-GATTEGNO Nata, 2005, De Corfou à Birkenau et à Jérusalem, Athènes : éd. Gavriilidis.

PINHAS Lisa, 2016, Récit de l'enfer, préface de Zanet Battinou, introduction d'Odette Varon-Vassard, avant-propos de Garyfallia Micha, Paris : Fondation pour la Mémoire de la Shoah, éditions Le Manuscrit, Collection « Témoignages de la Shoah».

TODOROv Tzvetan, 1994, Face à l'extrême (nouvelle édition), Paris : éd. du Seuil.

SEMPRÚN Jorge, 1996, l'Écriture ou la Vie, Paris : Gallimard, « Folio ».

SÉPHIHA André, 2010, Souvenirs d'une vie et d'un monde, Thessalonique : éd. Ianos.

SEVILlias Errikos, 2000, Athènes-Auschwitz, Athènes : éd. Hestia.

TILLION Germaine, 1988, Ravensbrück, Paris : Le Seuil.

VARON-VASSARD Odette, 2012, l'Émergence d'une mémoire difficile : essais sur le génocide des Juifs, $2^{\mathrm{e}}$ éd. augmentée en 2013, Athènes : Hestia (en grec).

VARON-VASSARD Odette, 2015, « le Génocide des Juifs de Grèce : histoire, mémoire, et historiographie », in Gérard DÉDÉYAN, Carol IANCU (dir.), Du génocide des Arméniens à la Shoah, Toulouse : Privat, p. 357-380.

VARON-VASSARD Odette, 2009, le Passage à l'âge adulte d'une génération : jeunes hommes et jeunes femmes sous l'Occupation et dans la Résistance, Athènes : Hestia (en grec).

Young people in the Maelstrom of occupied Greece: The persecution and Holocaust of the Jewish people 1943-1944, 2009, introduction d'Ariella ASER, Steven BOWMAN, Odette VARON-VASSARD, Athens: Central board of Jewish Communities in Greece.

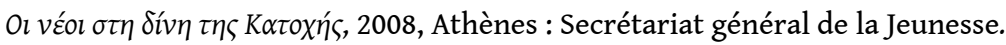

WIEVIORKA Annette, 2013, l'Ère du témoin, Paris : Libraire Arthème Fayard, 2013 [1 ${ }^{\text {re }}$ éd. 1998, Paris : éd. Plon].

\section{NOTES}

1. Texte remanié de la communication à l'Inalco, au Colloque «Témoignages dans les Balkans pendant les années $40 »$ (Paris, 22-23 novembre 2013). Je voudrais tout d'abord remercier le comité d'organisation de ce colloque, et spécialement Sophie Vassilaki, 
Joëlle Dalègre et Christina Alexopoulou, qui nous accueillent à l'Inalco, ainsi que les collègues grecques Ioanna Papathanassiou et Maria Thanopoulou (Centre national de recherche sociale en Grèce, EKKE), qui nous ont donné encore une fois la possibilité de nous rencontrer et de multiplier les échanges.

2. Voir VARON-VASSARD, 2012, l'Émergence d'une mémoire difficile: essais sur le génocide des Juifs; voir VARON-VASSARD, 2015, « le Génocide des Juifs de Grèce : histoire, mémoire, et historiographie ", in DÉDÉYAN Gérard, IANCU Carol (dir.), Du génocide des Arméniens à la Shoah, p. 357-380.

3. Tzvetan TODOROv, 1994, Face à l'extrême.

4. Jorge SEMPRÚN, 1996, l'Écriture ou la vie.

5. Hannah ARENDT, 1997, Eichmann à Jérusalem.

6. Annette WIEVIORKA, 2013, l'Ère du témoin, p. 81.

7. Miriam NOVITCH, 1982, le Passage des barbares : contribution à l'histoire de la déportation et de la résistance des Juifs de Grèce. La traduction en grec a été effectuée par Yorgos Zografakis (édition de l'Association pour l'Amitié gréco-israélienne, Athènes, 1985).

8. Rika BENVENISTE, 2014, Ceux qui ont survécu : résistance, déportation, retour, Athènes : Ed. Polis (en grec).

9. La recherche historique a été effectuée par Jason Chandrinos; responsable de l'exposition et la directrice du Musée Juif de Grèce, Zanet Battinou.

10. À Paris, il a été projeté à la soirée organisée par la délégation permanente de la Grèce auprès de l'Unesco, soirée organisée pour le jour de la mémoire de la Shoah (27 janvier 2014, à l'initiative de l'ambassadeur Catherine Daskalaki).

11. Boris CYRULNIK, 2010, Je me souviens..., et du même auteur 2012, Sauve-toi, la vie t'appelle.

12. SEMPRÚN, 1996, l'Écriture ou la Vie, p. 260.

13. Ce travail est lié à l'activité de Franguiski Abatzopoulou, professeur à l'Université de Thessalonique, qui a entrepris d'établir l'édition de plusieurs de ces témoignages.

14. Erika KOUNIO-AMARIGLIO, Albertos NAR, 1998, Témoignages oraux des Juifs de Salonique sur la Shoah. Ce recueil a été publié sous les soins de Franguiski Abatzopoulou.

15. André SÉPHIHA, 2010, Souvenirs d'une vie et d'un monde.

16. Berry nAHMiAs, 1989, Cri pour le lendemain. Ce livre, un des premiers témoignages en Grèce, soutenu par des présentations de Berry Nahmias elle-même, a connu beaucoup de succès et de nombreuses rééditions, mais comme elles n'ont pas été signalées par l'éditeur on n'en a pas de traces.

17. Errikos SEVILLIAS, 2000, Athènes-Auschwitz.

18. Nata OSMO-GATTEGNO, 2005, De Corfou à Birkenau et à Jérusalem.

19. Édition anglophone : CENTRAL BOARD OF JEWISH COMMUNITIES IN GREECE, 2009, Young people in the Maelstrom of occupied Greece: The persecution and Holocaust of the Jewish people 1943-1944, introductive texts by Ariella ASER, Steven BOWMAN, Odette VARON-VASSARD,

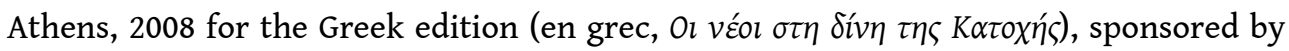
the General Secretariat of Youth. 
20. Lisa PINHAS, 2014, A Narrative of evil: Lisa Pinhas confronts the Holocaust. La transcription du manuscrit en français et la traduction du français en grec ont été

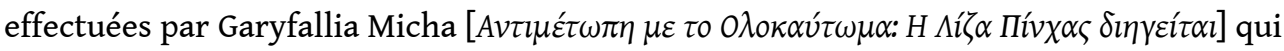
a aussi annoté le texte de plus de 200 notes. L'édition grecque a été publiée en même temps que la version anglaise. Les deux versions sont accompagnées d'une introduction de Gabriella Etmektsoglou. L'édition française de la version originale publiée en juillet 2016 : Lisa PINHAS, Récit de l'enfer, préface de Zanet Battinou, introduction d'Odette Varon-Vassard, avant-propos de Garyfallia Micha, Paris : Fondation pour la Mémoire de la Shoah, éditions Le Manuscrit, Collection « Témoignages de la Shoah».

21. Charlotte DELBO, 1961, le Convoi du 24 Janvier.

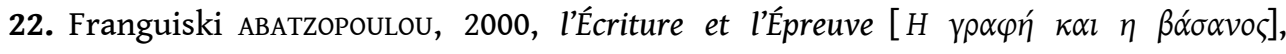
p. 286-337. Les pages mentionnées ont été publiées pour la première fois dans la revue féminine Dini $[\Delta i v \eta]$ en 1997, sous le titre : «le génocide et la parole féminine ». Voir aussi Germaine TILLION, 1988, Ravensbrück.

23. Rappelons qu'il s'agit de la communauté d'origine de l'écrivain Albert COHEN, qui se réfère longuement à elle surtout dans ses romans des années 1930 (Solal, 1930 et Mangeclous, 1938).

24. Les initiales signifient "Organisation Unifiée Panhellénique des Jeunes", la plus grande organisation de jeunes, créée en février 1943, à l'initiative des organisations de la gauche, mais fonctionnant comme un pôle d'attraction pour une énorme partie de la jeune génération. Voir VARON-VASSARD, 2009, le Passage à l'âge adulte d'une génération: jeunes hommes et jeunes femmes sous l'Occupation et dans la Résistance (en grec).

25. En ce début 2015 elle est toujours vivante en Israël et active.

26. Primo LeVI, 1987, Si c'est un homme, p. 9.

27. Robert ANTELME, 1957, l'Espèce humaine.

28. LEVI, 2003, la Trêve.

29. Appartenant à un ordre armé, connus comme germanotsoliades et en tant que collaborateurs des Allemands ils ont perpétré maints crimes et massacres entre les Grecs.

\section{RÉSUMÉS}

Dans cet article nous posons d'abord la question du silence sur le génocide des Juifs et l'expérience des camps d'extermination nazis et la lente émergence de cette mémoire, en faisant un survol bibliographique dans la bibliographie grecque.

Ensuite nous focalisons sur des sujets qui ont à voir avec la survie de jeunes femmes grecques juives au camp d'Auschwitz-Birkenau. Le corpus des témoignages auquel nous nous référons comporte les livres suivants : Lisa Pinhas, Récit de l'enfer, publié en français en juillet 2016 par la Fondation pour la Mémoire de la Shoah, Nata Osmo-Gattegno, De Corfou à Birkenau et à Jérusalem (en grec), Berry Nahmias, Cri pour le lendemain (en grec), Erika Kounio-Amariglio, Pour que le monde entier sache: Thessalonique-Auschwitz et retour (édition en français). 
Nous traitons des sujets tels que la condition féminine des déportées, les relations avec les autres déportées, la solidarité, la sociabilité, le sentiment d'identité nationale. Certains de ces paramètres ont été cruciaux pour leur survie.

This article first raises the issue of the silence about the genocide of the Jews, the experience of the Nazi extermination camps and the slow emergence of the memory, by proceeding through a review of the relevant Greek bibliography. Then, focus is placed on issues related to the survival of young Greek Jewish women at the camp of Auschwitz-Birkenau. The corpus of testimonies to which reference is made, consists of the following books: A Narrative of Evil, Lisa Pinhas confronts the Holocaust (transcription, translation in Greek Garyfallia Micha), Nata Osmo-Gattegno, From Corfu to Birkenau and Jerusalem, Berry Nahmias, A Cry for Tomorrow, Erika Kounio-Amariglio, Fifty years later... Memories of a Salonica Jewish woman. We address issues such as the condition of female detention, relations among the inmates, solidarity, sociability, sense of national identity. Some of these parameters have been pivotal to their survival.

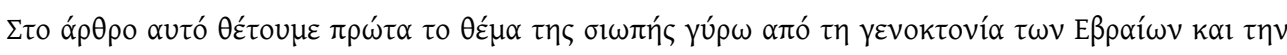

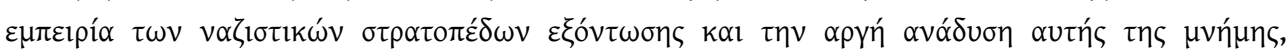

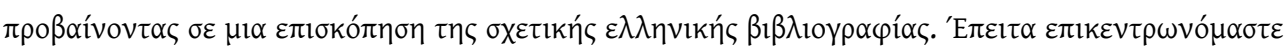

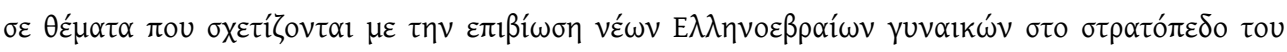

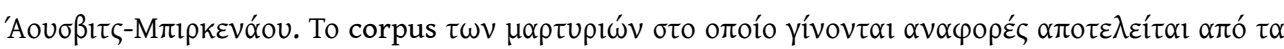

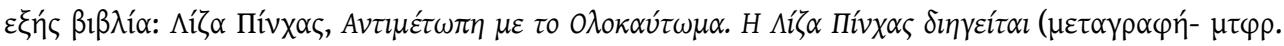

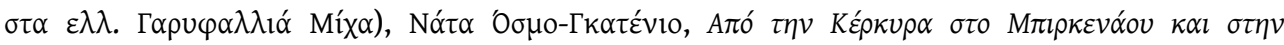

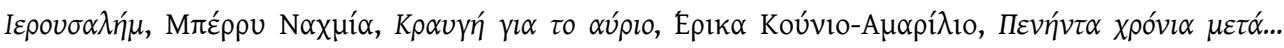

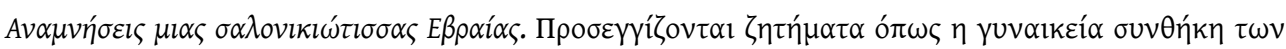

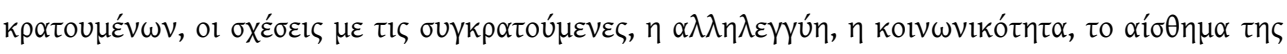

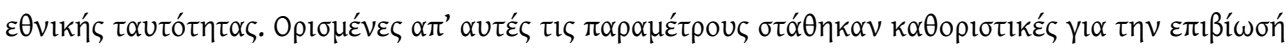

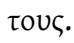

\section{INDEX}

Index géographique : Grèce

motsclesmk ЕВРЕИТЕ, ДЕПОРТАЦИЈА, ГРЦИЈА, ВТОРАТА СВЕТСКА ВОЈНА, ИСТОРИЈА, СВЕДОШТВА

Mots-clés : déportation, déportation, Juifs, Juifs

motsclestr Yahudi, Sürgün, Yunanistan, İkinci Dünya Savaşı, Tarih, Tanıklar

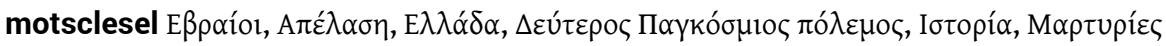

Thèmes : Histoire

Keywords : Deportation, Jews, Greece, WWII, History, Testimonies

Index chronologique : guerre mondiale (1939-1945)

\section{AUTEUR}

\section{ODETTE VARON-VASSARD}

Université grecque ouverte 